\title{
On the Convergence of Non-Polynomial Spline Finite Difference Method for Quasi-Linear Elliptic Boundary Value Problems in Two-Space Dimensions
}

\author{
Navnit Jha ${ }^{{ }^{*}}$, Ravindra Kumar ${ }^{2}$, R. K. Mohanty ${ }^{1}$ \\ ${ }^{1}$ Department of Mathematics, South Asian University, Chanakyapuri, New Delhi 110 021, India \\ ${ }^{2}$ Department of Mathematics, Rajdhani College, University of Delhi, Delhi 110 015, India. \\ Email: navnitjha@sau.ac.in
}

\begin{abstract}
An approximate solution technique based on non-polynomial spline and finite difference method has been described for two-space dimensional quasi-linear elliptic boundary value problems. The numerical scheme in the limiting case of non-polynomial spline parameter provides the cubic spline scheme. The proposed scheme is analyzed for the convergence using matrix theory. Experimental results show the importance of using non-polynomial spline scheme over corresponding cubic spline scheme in terms of iterations to achieve desired accuracy. The method is tested for the convergence and corroborates the theoretical truncation errors. The accuracy in the solutions is obtained for Tricomi and KhokhlovZabolotskaya equations for various mesh step sizes.
\end{abstract}

Keywords: Finite difference method, Non-polynomial spline, Tricomi equation, Khokhlov-Zabolotskaya equation, Maximum absolute errors.

\section{Introduction}

In this paper, a new numerical method for the numerical solution of quasi-linear elliptic boundary value problems (EBVPs) with significant first order partial derivatives is presented. The proposed equation possesses the following form

$$
A(x, y, U) \partial_{x}^{2} U+B(x, y, U) \partial_{y}^{2} U=G\left(x, y, U, \partial_{x} U, \partial_{y} U\right),(x, y) \in \Omega
$$

where the values of $U(x, y)$ at the boundary $\partial \Omega$ are known to us and the symbol $\partial_{z}^{n} U$ stands for $\partial^{n} U / \partial z^{n}, z=x, y$.

Our aim is to obtain the accurate numerical values of the unknown faction $U(x, y)$ on the domain of integration $\Omega$ by means of discrete approximations based on finite Taylor's expansion and nonpolynomial spline having hyperbolic functions as basis elements. The integration of (1) to the arbitrary choice of $A(x, y, U), B(x, y, U)$ and/or $G(x, y, U)$ is difficult and thus, it is essential to develop an efficient numerical method.

Such EBVPs frequently occur in combustion theory, plasma physics, steady state heat and mass transfer equation with volume reaction, steady transonic gas flow, mass transfer with a volume chemical reaction in translational shear fluid flow, stationary anisotropic diffusion equation etc. (see e.g. $[1,5,10,20,22])$. In the context of EBVPs, researchers have developed approximation techniques using collocation spline, finite element method, finite difference method, radial basis functions and wavelet method (see $[4,16,24,27,28]$ ). In particular, the Legendre and Haar wavelets have been applied to linear EBVPs by Aziz et al. [2]. A combined approach to radial basis functions and collocation method has been developed to solve EBVPs with Dirichlet-Neumann boundary values by $\mathrm{Hu}[9]$. With the various known numerical techniques, the finite difference method is a beautiful tool that efficiently and accurately computes the solution values to the wide range of differential systems (LeVeque [17] and Zhao 
et al. [30]). A non-polynomial spline method using the basis $\{\sin (\tau x), \cos (\tau x), x, 1\}$ for one-dimensional hyperbolic equations was described by Rashidinia et al. [23]. A tension spline method with exponential functions as a basis element to time dependent one-dimensional singular partial differential equations has been considered by Gopal et al. [6]. Islam et al. [25] have constructed a non-polynomial spline technique to solve third order obstacle problems. The hyperbolic spline and quintic spline basis for two-point boundary value problems has been discussed by Jha et al. [11, 12]. The implementation of parametric spline to the boundary value problems has been discussed by Khan [13]. In the current literature survey, we have limited articles on the extension of spline with trigonometric, exponential or hyperbolic functions for the numerical solution of higher dimensional partial differential equations.

The central difference operator and averaging operators giving second order of compact scheme proves to be good for the regular EBVPs, the method usually deteriorates in case of quasi-linear problems, thus it is customary to devise a higher order method. Thus, in this article, our attempt is to obtain an $O\left(k^{2}+h^{2} k^{2}+h^{4}\right)$-accurate algorithm that implements non-polynomial spline basis in $x$-directions and parallel to $\mathrm{x}$-axis at each discrete grid point on $y$-axis. The method is developed in combination with finite difference approximations on nine point stencils. The proposed method is compact and therefore, the discrete system of equations can be easily solved.

\section{Non-Polynomial Spline Interpolation}

The consideration of non-polynomial spline for obtaining the solution of EBVPs compensates the loss of smoothness inherited by corresponding polynomial cubic spline. The non-polynomial spline function considered here has the basis $\mathcal{B}=\{\sinh (\omega x), \cosh (\omega x), x, 1\}$, where $\omega$ is a finite real number denoting the frequency of hyperbolic functions. The usual cubic spline basis $\mathcal{B}^{*}=\left\{x^{3}, x^{2}, x, 1\right\}$ can be easily obtained from the hyperbolic spline basis $\mathcal{B}$ as a limiting case $\omega \rightarrow 0$, and it is evident from the spanning relation $\operatorname{span}[\mathcal{B}]=\operatorname{span}\left[\left\{6(\sinh (\omega x)-\omega x) / \omega^{3}, 2(\cosh (\omega x)-1) / \omega^{2}, x, 1\right\}\right]=\operatorname{span}\left[\mathcal{B}^{*}\right]$. Let $\Omega_{h, k}=\left\{\left(x_{l}, y_{m}\right): x_{l}=a+l h, y_{m}=c+m k, l=1(1) L+1, m=1(1) M+1\right\}$ be the rectangular network, as a uniform mesh partition of the solution domain $\Omega \cup \partial \Omega=[a, b] \times[c, d]$, where $h=(b-a) /(L+1)$ and $k=(d-c) /(M+1), L, M \in \mathbb{Z}_{+}$. Let $\lambda=k / h>0$ be the constant mesh ratio parameter and $u_{l, m}$ represent the numerical solution values, while $U_{l, m}$ be the exact value of $U(x, y)$ at the mesh point $\left(x_{l}, y_{m}\right)$.

Let us denote $\mathcal{S}_{m}(x)$ as the hyperbolic spline interpolating non-polynomial along $x$-direction on $\left[x_{l-1}, x_{l}\right], l=1(1) L+1$, which is defined as follows

$$
\mathcal{S}_{m}(x)=a_{l} \sinh \left(\omega\left(x-x_{l}\right)\right)+b_{l} \cosh \left(\omega\left(x-x_{l}\right)\right)+c_{l}\left(x-x_{l}\right)+d_{l}
$$

and satisfies at $m^{\text {th }}, m=0(1) M+1$ line parallel to $x$-axis, the following properties:

$$
\begin{aligned}
\mathcal{S}_{m}\left(x_{l}\right)=U_{l, m}, & \mathcal{S}_{m}\left(x_{l-1}\right)=U_{l-1, m}, \\
\left(\frac{d^{2} \mathcal{S}_{m}(x)}{d x^{2}}\right)_{x_{l}}=\mathcal{M}_{l, m}, & \left(\frac{d^{2} \mathcal{S}_{m}(x)}{d x^{2}}\right)_{x_{l-1}}=\mathcal{M}_{l-1, m}
\end{aligned}
$$

With the help of algebraic calculations, one obtains 


$$
\begin{gathered}
a_{l}=\left[\cosh (\theta) \mathcal{M}_{l, m}-\mathcal{M}_{l-1, m}\right] /\left[\omega^{2} \sinh (\theta)\right], \quad b_{l}=\mathcal{M}_{l, m} / \omega^{2}, \\
c_{l}=\left[\left(U_{l, m}-U_{l-1, m}\right) \omega^{2}-\left(\mathcal{M}_{l, m}-\mathcal{M}_{l-1, m}\right)\right] /[\omega \theta], \quad d_{l}=\left[\omega^{2} U_{l, m}-\mathcal{M}_{l, m}\right] / \omega^{2},
\end{gathered}
$$

where $\theta=\omega h$.

After substituting the expressions of $a_{l}, b_{l}, c_{l}$ and $d_{l}$ in (2), we can obtain the spline relation on $\left[x_{l-1}, x_{l}\right], l=1(1) L+1$.

In a similar manner, the interpolating non-polynomial on $\left[x_{l}, x_{l+1}\right], l=0(1) L$ is given by

$$
\begin{aligned}
\mathcal{S}_{m}\left(x_{l}\right)= & \frac{\left(\mathcal{M}_{l+1, m} \cosh (\theta)-\mathcal{M}_{l, m}\right) \sinh \left(\omega\left(x-x_{l}\right)\right)}{\omega^{2} \sinh (\theta)}+\frac{\mathcal{M}_{l+1, m} \cosh \left(\omega\left(x-x_{l}\right)\right)}{\omega^{2}} \\
& +\frac{\left[\left(U_{l+1, m}-U_{l, m}\right) \omega^{2}+\mathcal{M}_{l, m}-\mathcal{M}_{l+1, m}\right]\left(x-x_{l}\right)}{\omega \theta}+U_{l+1, m}-\frac{\mathcal{M}_{l+1, m}}{\omega^{2}}
\end{aligned}
$$

where

$$
\mathcal{S}_{m}\left(x_{l}\right)=U_{l, m}, \mathcal{S}_{m}\left(x_{l+1}\right)=U_{l+1, m},\left(\frac{d^{2} \mathcal{S}_{m}(x)}{d x^{2}}\right)_{x_{l}}=\mathcal{M}_{l, m},\left(\frac{d^{2} \mathcal{S}_{m}(x)}{d x^{2}}\right)_{x_{l+1}}=\mathcal{M}_{l+1, m}
$$

The spline relations (2) and (3) will be used to obtain high order approximations at the fictitious node $\left(x_{l \pm \eta}, y_{m}\right), 0<\eta<1$. The continuity of first order partial derivatives with respect to $x$ gives us second order accuracy and therefore is left for the discussion.

\section{Finite Difference Approximations}

Initially, we obtain compact discretization to the linear EBVPs with variable coefficients

$$
A(x, y) \partial_{x}^{2} U+B(x, y) \partial_{y}^{2} U=G(x, y),(x, y) \in \Omega
$$

that does not involve independent variable $U(x, y)$ and its first order partial derivatives as a non-linear term. Later the method will be extended by means of non-polynomial spline to obtain approximations of quasi-linear equation (1). We need following approximations

$$
\begin{gathered}
\tilde{U}_{l \pm \eta, m}=\left[\eta(\eta \pm 1) U_{l+1, m}-2\left(\eta^{2}-1\right) U_{l, m}+\eta(\eta \mp 1) U_{l-1, m}\right] / 2 \\
\tilde{U}_{l \pm \eta, m+1}=\left[\eta(\eta \pm 1) U_{l+1, m+1}-2\left(\eta^{2}-1\right) U_{l, m+1}+\eta(\eta \mp 1) U_{l-1, m+1}\right] / 2 \\
\tilde{U}_{l \pm \eta, m-1}=\left[\eta(\eta \pm 1) U_{l+1, m-1}-2\left(\eta^{2}-1\right) U_{l, m-1}+\eta(\eta \mp 1) U_{l-1, m-1}\right] / 2 \\
\tilde{U}_{y y_{l, m}}=\left[U_{l, m+1}-2 U_{l, m}+U_{l, m-1}\right] / k^{2} \\
\tilde{U}_{y y_{l \eta, m}}=\left[\tilde{U}_{l \pm \eta, m+1}-2 \tilde{U}_{l \pm \eta, m}+\tilde{U}_{l \pm \eta, m-1}\right] / k^{2}
\end{gathered}
$$

Following the idea of Numerov's method (see Lambert [15]), the finite difference replacement of linear EBVPs (4) with variable coefficients is given by 


$$
\begin{aligned}
{\left[A_{l, m}-\frac{h^{2}}{6} \frac{A_{x_{l, m}}^{2}}{A_{l, m}}+\frac{h^{2}}{12} A_{x x_{l, m}}\right] } & \delta_{x}^{2} U_{l, m}=\frac{h^{2}}{12 \eta^{2}}\left[\left(1-\frac{\eta h A_{x_{l, m}}}{A_{l, m}}\right)\left(G_{l+\eta, m}-B_{l+\eta, m} \tilde{U}_{y y_{l+\eta, m}}\right)\right. \\
& \left.+\left(12 \eta^{2}-2\right)\left(G_{l, m}-B_{l, m} \tilde{U}_{y l_{l, m}}\right)+\left(1+\frac{\eta h A_{x_{l, m}}}{A_{l, m}}\right)\left(G_{l-\eta, m}-B_{l-\eta, m} \tilde{U}_{y y_{l-\eta, m}}\right)\right] \\
& +O\left(k^{2} h^{2}+k^{2} h^{4}+h^{6}\right)
\end{aligned}
$$

where $\delta_{x}$ is the central difference operator.

Now, we extend the method (10), to the two-space dimensional EBVPs involving first order partial derivatives:

$$
A(x, y) \partial_{x}^{2} U+B(x, y) \partial_{y}^{2} U=G\left(x, y, U, \partial_{x} U, \partial_{y} U\right),(x, y) \in \Omega
$$

and obtain compatible order approximation on the nine point compact stencils. We need to consider some approximations to the derivatives:

$$
\begin{gathered}
\tilde{U}_{x_{l, m}}=\left[U_{l+1, m}-U_{l-1, m}\right] /[2 h] \\
\tilde{U}_{x_{l \pm 1, m}}=\left[ \pm 3 U_{l \pm 1, m} \mp 4 U_{l, m} \pm U_{l \mp 1, m}\right] /[2 h] \\
\tilde{U}_{y_{l, m}}=\left[U_{l, m+1}-U_{l, m-1}\right] /[2 k] \\
\tilde{U}_{y_{l \pm 1, m}}=\left[U_{l \pm 1, m+1}-U_{l \pm 1, m-1}\right] /[2 k] \\
\tilde{U}_{y y_{l+1, m}}=\left[U_{l \pm 1, m+1}-2 U_{l \pm 1, m}+U_{l \pm 1, m-1}\right] / k^{2}
\end{gathered}
$$

Define

$$
\begin{gathered}
\tilde{G}_{l, m}=G\left(x_{l}, y_{m}, U_{l, m}, \tilde{U}_{x_{l, m}}, \tilde{U}_{y_{l, m}}\right) \\
\tilde{G}_{l \pm 1, m}=G\left(x_{l \pm 1}, y_{m}, U_{l \pm 1, m}, \tilde{U}_{x_{l \pm 1, m}}, \tilde{U}_{y_{l \pm 1, m}}\right)
\end{gathered}
$$

Incorporating the approximations (12)-(16) in its expanded forms to the equations (17)-(18), we find

$$
\begin{gathered}
\tilde{G}_{l, m}=G_{l, m}+\frac{h^{2}}{6} a_{l, m} U_{x x x l, m}+O\left(h^{4}+k^{2}\right) \\
\tilde{G}_{l \pm 1, m}=G_{l, m}-\frac{h^{2}}{3} a_{l, m} U_{x x x l, m} \mp \frac{h^{3}}{12}\left(a_{l, m} U_{x x x x l, m}+4 a_{x l, m} U_{x x x l, m}\right)+O\left(h^{4}+k^{2}\right)
\end{gathered}
$$

where $a_{l, m}=\left(\partial_{u_{x}}^{4} G(\bullet)\right)_{l, m}$ etc.

Let

$$
\begin{aligned}
& \tilde{\mathcal{M}}_{l, m}=\frac{1}{A_{l, m}}\left(\tilde{G}_{l, m}-B_{l, m} \tilde{U}_{y y_{l, m}}\right) \\
& \tilde{\mathcal{M}}_{l \pm 1, m}=\frac{1}{A_{l, m}}\left(1 \mp \frac{h A_{x_{l, m}}}{A_{l, m}}\right)\left(\tilde{G}_{l \pm 1, m}-B_{l \pm 1, m} \tilde{U}_{y y_{l \pm 1, m}}\right)
\end{aligned}
$$

Now, the additional approximation

$$
\hat{U}_{x_{l, m}}=\tilde{U}_{x_{l, m}}-\frac{h}{12}\left(\tilde{\mathcal{M}}_{l+1, m}-\tilde{\mathcal{M}}_{l-1, m}\right)
$$

gives us $\hat{U}_{x_{l, m}}=U_{x_{l, m}}+O\left(h^{4}+k^{2}\right)$.

With the help of non-polynomial spline relations (2) and (3), we construct a new approximation to the solution values and its $\mathrm{x}$-derivative at the fictitious node $\left(x_{l \pm \eta}, y_{m}\right), 0<\eta<1$, as follows: 


$$
\begin{gathered}
\hat{U}_{l \pm \eta, m}=\eta U_{l \pm 1, m}+(1-\eta) U_{l, m}+\frac{(\eta-1) \sinh (\theta)-\sinh ((\eta-1) \theta)}{\omega^{2} \sinh (\theta)} \tilde{\mathcal{M}}_{l, m} \\
+\frac{\sinh (\eta \theta)-\eta \sinh (\theta)}{\omega^{2} \sinh (\theta)} \tilde{\mathcal{M}}_{l \pm 1, m} \\
\hat{U}_{x_{l \pm \eta, m}}= \pm \frac{1}{h}\left(U_{l \pm 1, m}-U_{l, m}\right) \pm \frac{\theta \cosh (\eta \theta)-\sinh (\theta)}{h \theta \sinh (\theta)} \tilde{\mathcal{M}}_{l \pm 1, m} \\
\pm \frac{\sinh (\theta)-\theta \cosh ((\eta-1) \theta)}{\omega \theta \sinh (\theta)} \tilde{\mathcal{M}}_{l, m}
\end{gathered}
$$

With the application of series expansions, it is easy to see that

$$
\begin{aligned}
\hat{U}_{l \pm \eta, m}= & U_{l \pm \eta, m}+O\left(h^{4}+k^{2}\right) \\
\hat{U}_{x_{l \pm \eta, m}}= & U_{x_{l \pm \eta, m}} \mp \frac{h^{3}}{12} \eta(3 \eta-2) a_{l, m} U_{x x x l, m} \pm \frac{h^{3}}{24}(2 \eta-1)\left(2 \eta^{2}-2 \eta-1\right)\left(\omega^{2} U_{x x l, m}-U_{x x x x l, m}\right) \\
& \quad+O\left(h^{4}+k^{2}\right)
\end{aligned}
$$

Now, consider the new functional approximations

$$
\begin{gathered}
\hat{G}_{l, m}=G\left(x_{l}, y_{m}, U_{l, m}, \hat{U}_{x_{l, m}}, \tilde{U}_{y_{l, m}}\right) \\
\hat{G}_{l \pm \eta, m}=G\left(x_{l \pm \eta}, y_{m}, \hat{U}_{l \pm \eta, m}, \hat{U}_{x_{l \pm \eta, m}}, \tilde{U}_{y_{l \pm \eta, m}}\right)
\end{gathered}
$$

Incorporating the equations (14), (22) and (25)-(27) and applying the series expansions, we find

$$
\begin{gathered}
\hat{G}_{l, m}=G_{l, m}+O\left(h^{4}+k^{2}\right) \\
\hat{G}_{l \pm \eta, m}=G_{l \pm \eta, m} \pm \frac{h^{3}}{6} \eta\left(1-\eta^{2}\right) B_{l, m} U_{x x x l, m}+O\left(h^{4}+k^{2}\right)
\end{gathered}
$$

Following the concept of cubic spline (Mohanty et al. [18]), and in view of the relations (28) and (29), the non-polynomial spline finite difference method for the numerical estimation of solution values to the equation (11) is given by the formula

$$
\begin{aligned}
{\left[A_{l, m}-\frac{h^{2}}{12}\left(\frac{2 A_{x_{l, m}}^{2}}{A_{l, m}}-A_{x x_{l, m}}\right)\right] } & \delta_{x}^{2} U_{l, m}=\frac{h^{2}}{12 \eta^{2}}\left[\left(1-\frac{\eta h A_{x_{l, m}}}{A_{l, m}}\right)\left(\hat{G}_{l+\eta, m}-B_{l+\eta, m} \tilde{U}_{y y_{l+\eta, m}}\right)\right. \\
& \left.+\left(12 \eta^{2}-2\right)\left(\hat{G}_{l, m}-B_{l, m} \tilde{U}_{y y_{l, m}}\right)+\left(1+\frac{\eta h A_{x_{l, m}}}{A_{l, m}}\right)\left(\hat{G}_{l-\eta, m}-B_{l-\eta, m} \tilde{U}_{3 y_{l-\eta, m}}\right)\right] \\
& +\hat{T}_{l, m}
\end{aligned}
$$

where local truncation error of the scheme (30) is estimated as

$$
\hat{T}_{l, m}=O\left(h^{2} k^{2}+h^{2} k^{4}+h^{4} k^{2}+h^{6}\right)
$$

and thus, we have achieved an $h^{-2} \hat{T}_{l, m}=O\left(k^{2}+h^{2} k^{2}+h^{4}\right)$-accurate method for the non-linear EBVPs (11).

The scheme (30) is compact and free from the fictitious values of unknown function $U(x, y)$. The nonpolynomial spline scheme (30) as $\omega \rightarrow 0$ and $\eta=1$, gives us a particular case of cubic spline scheme developed by Mohanty et al. [19]. 
In addition, if $A=A(x, y, U)$ and $B=B(x, y, U)$ in equation (11), then the substitution $A_{x_{l, m}}=\left(\mu_{x} \delta_{x} A_{l, m}\right) / h$ and $A_{x x_{l, m}}=\left(\delta_{x}^{2} A_{l, m}\right) / h^{2}$, leads to the same local truncation error as shown in equation (31), where $\mu_{x}$ is the averaging difference operator. Thus, a minor modification to the scheme (30) leads to nine-point compact scheme to quasi-linear EBVPs (1). The resulting system of discrete equations must be solved in combination with the boundary conditions and on neglecting higher order terms in equation (30).

\section{Convergence Analysis and Bounds of Error}

In this section, we obtain bounds on the solution error and thereby establish the convergence criterion. It is essential to show that error obtained in the numerical solution should tend to zero as the mesh step sizes becomes sufficiently small. Moreover, the EBVPs (11) to be elliptic, if both $A(x, y)>0, B(x, y)>0$ or $A(x, y)<0, B(x, y)<0$, thus without loss of generality, we assume that $A(x, y)>0$ and $B(x, y)>0$, in the following analysis. The convergence analysis in the case that both $A(x, y)$ and $B(x, y)$ are negative follows in a similar manner. The EBVPs (11), at the mesh point $\left(x_{l}, \mathscr{y}_{m}\right)$, $l=1(1) L, m=1(1) M$, can be written as:

$$
A\left(x_{l}, y_{m}\right)\left(\partial_{x}^{2} U\right)_{\left(x_{l}, y_{m}\right)}+B\left(x_{l}, y_{m}\right)\left(\partial_{y}^{2} U\right)_{\left(x_{l}, y_{m}\right)}=G\left(x_{l}, y_{m}, U_{l, m},\left(\partial_{x} U\right)_{\left(x_{l}, y_{m}\right)},\left(\partial_{y} U\right)_{\left(x_{l}, y_{m}\right)}\right)
$$

Then, the numerical method (30), may be represented by the recurrence relations

$$
\begin{aligned}
& -\lambda^{2}\left[A_{l, m}-\frac{h^{2}}{12}\left(\frac{2 A_{x_{l, m}}^{2}}{A_{l, m}}-A_{x x_{l, m}}\right)\right] \delta_{x}^{2} U_{l, m}+\frac{k^{2}}{12 \eta^{2}}\left[\left(1-\frac{\eta h A_{x_{l, m}}}{A_{l, m}}\right)\left(\hat{G}_{l+\eta, m}-B_{l+\eta, m} \tilde{U}_{y y_{l+\eta, m}}\right)\right. \\
& \left.+\left(12 \eta^{2}-2\right)\left(\hat{G}_{l, m}-B_{l, m} \tilde{U}_{y y_{l, m}}\right)+\left(1+\frac{\eta h A_{x_{l, m}}}{A_{l, m}}\right)\left(\hat{G}_{l-\eta, m}-B_{l-\eta, m} \tilde{U}_{y y_{l-\eta, m}}\right)\right]+T_{l, m}
\end{aligned}
$$

where $T_{l, m}=O\left(k^{4}+h^{2} k^{4}+h^{4} k^{2}\right)$.

Equivalently,

$$
\psi_{l, m}+T_{l, m}=0, l=1(1) L, m=1(1) M
$$

where $\psi_{l, m}$ is obtained from the equation (33).

The difference relation (34), in the matrix notation can be expressed as follows

$$
\boldsymbol{\psi}(\boldsymbol{U})+\boldsymbol{T}=0_{L M \times L M}
$$

where $\boldsymbol{T}=\left[T_{11}, T_{21}, \ldots, T_{L 1}, T_{12}, T_{22}, \ldots, T_{L 2}, \ldots, T_{1 M}, T_{2 M}, \ldots, T_{L M}\right]^{T}$ is the local truncation error vector with each $T_{l m}$ being $O\left(k^{4}+h^{2} k^{4}+h^{4} k^{2}\right)$ and $\boldsymbol{U}=\left[U_{11}, U_{21}, \ldots, U_{L 1}, U_{12}, U_{22}, \ldots, U_{L 2}, \ldots, U_{1 M}, U_{2 M}, \ldots, U_{L M}\right]^{\mathrm{T}}$ is the vector of solution values and $\boldsymbol{\psi}(\boldsymbol{U})=\left[\psi_{11}, \psi_{21}, \ldots, \psi_{L 1}, \psi_{12}, \psi_{22}, \ldots, \psi_{L 2}, \ldots, \psi_{1 M}, \psi_{2 M}, \ldots, \psi_{L M}\right]^{\mathrm{T}}$.

Our objective is to determine the approximation $\boldsymbol{u}$ for the exact solution values $\boldsymbol{U}$, which is obtained by solving the equation

$$
\boldsymbol{\psi}(\boldsymbol{u})=0_{L M \times L M}
$$

and therefore, with the help of equations (35) and (36), one obtains

$$
\boldsymbol{\psi}(\boldsymbol{u})-\boldsymbol{\psi}(\boldsymbol{U})=\boldsymbol{T}
$$


Let $\epsilon_{l, m}=u_{l, m}-U_{l, m}, l=1(1) L, m=1(1) M$ be the point-wise error and $\epsilon=\left[\epsilon_{11}, \epsilon_{21}, \ldots, \epsilon_{L 1}, \epsilon_{12}, \epsilon_{22}, \ldots\right.$, $\left.\epsilon_{L 2}, \ldots, \epsilon_{1 M}, \epsilon_{2 M}, \ldots, \epsilon_{L M}\right]^{\mathrm{T}}$ be the error vector.

Let us define

$$
\begin{aligned}
\tilde{\epsilon}_{l \pm \eta, m} & =\left[\eta(\eta \pm 1) \epsilon_{l+1, m}-2\left(\eta^{2}-1\right) \epsilon_{l, m}+\eta(\eta \mp 1) \epsilon_{l-1, m}\right] / 2 \\
\tilde{\epsilon}_{l \pm \eta, m+1} & =\left[\eta(\eta \pm 1) \epsilon_{l+1, m+1}-2\left(\eta^{2}-1\right) \epsilon_{l, m+1}+\eta(\eta \mp 1) \epsilon_{l-1, m+1}\right] / 2 \\
\tilde{\epsilon}_{l \pm \eta, m-1} & =\left[\eta(\eta \pm 1) \epsilon_{l+1, m-1}-2\left(\eta^{2}-1\right) \epsilon_{l, m-1}+\eta(\eta \mp 1) \epsilon_{l-1, m-1}\right] / 2 \\
\tilde{\epsilon}_{y y_{l \pm \eta, m}} & =\left[\tilde{\epsilon}_{l \pm \eta, m+1}-2 \tilde{\epsilon}_{l \pm \eta, m}+\tilde{\epsilon}_{l \pm \eta, m-1}\right] / k^{2} \\
\tilde{\epsilon}_{y y_{l, m}} & =\left[\epsilon_{l, m+1}-2 \epsilon_{l, m}+\epsilon_{l, m-1} / k^{2}\right. \\
\tilde{\epsilon}_{x_{l, m}} & =\left[\epsilon_{l+1, m}-\epsilon_{l-1, m}\right] /[2 h] \\
\tilde{\epsilon}_{x_{l \pm 1, m}} & =\left[ \pm 3 \epsilon_{l \pm 1, m} \mp 4 \epsilon_{l, m} \pm \epsilon_{l \mp 1, m}\right] /[2 h] \\
\tilde{\epsilon}_{y_{l, m}} & =\left[\epsilon_{l, m+1}-\epsilon_{l, m-1}\right] /[2 k] \\
\tilde{\epsilon}_{y_{l \pm 1, m}} & =\left[\epsilon_{l \pm 1, m+1}-\epsilon_{l \pm 1, m-1}\right] /[2 k] \\
\tilde{\epsilon}_{y y_{l \pm 1, m}} & =\left[\epsilon_{l \pm 1, m+1}-2 \epsilon_{l \pm 1, m}+\epsilon_{l \pm 1, m-1}\right] / k^{2}
\end{aligned}
$$

For $\delta=0,1$, we define

$$
\begin{aligned}
& \tilde{g}_{l \pm \delta, m}=G\left(x_{l \pm \delta}, y_{m}, u_{l \pm \delta, m}, \tilde{u}_{x_{l \pm \delta, m}}, \tilde{u}_{y_{l \pm \delta, m}}\right) \approx \tilde{G}_{l \pm \delta, m} \\
& \tilde{E}_{l \pm \delta, m}=\tilde{g}_{l \pm \delta, m}-\tilde{G}_{l \pm \delta, m}
\end{aligned}
$$

Let

$$
\begin{gathered}
\tilde{\mathcal{M}}_{l \pm \delta, m}^{\epsilon}=\frac{1}{A_{l, m}}\left(1 \mp \frac{\delta h A_{x_{l, m}}}{A_{l, m}}\right)\left(\tilde{E}_{l \pm \delta, m}-B_{l \pm \delta, m} \tilde{\epsilon}_{y y_{l \pm \delta, m}}\right) \\
\hat{\epsilon}_{x_{l, m}}=\tilde{\epsilon}_{x_{l, m}}-\frac{h}{12}\left(\tilde{\mathcal{M}}_{l+1, m}^{\epsilon}-\tilde{\mathcal{M}}_{l-1, m}^{\epsilon}\right)
\end{gathered}
$$

Replacing the symbols $U_{l \pm \eta, m}$ and $\mathcal{M}_{l \pm \eta, m}$ by $\epsilon_{l \pm \eta, m}$ and $\hat{\mathcal{M}}_{l \pm \eta, m}^{\epsilon}$ respectively in equations (23)-(24), we can easily obtain the expressions of $\hat{\epsilon}_{l \pm \eta, m}$ and $\hat{\epsilon}_{x_{l \pm \eta, m}}$ in a similar manner. Thereby, applying the Mean value theorem, one obtains

$$
\hat{E}_{l \pm \delta \eta, m}=\hat{a}_{l \pm \delta \eta, m} \hat{\epsilon}_{x_{l \pm \delta \eta, m}}+\hat{b}_{l \pm \delta \eta, m} \hat{\epsilon}_{y_{l \pm \delta \eta, m}}+\hat{c}_{l \pm \delta \eta, m} \hat{\epsilon}_{l \pm \delta \eta, m}, \quad \delta=0,1
$$

for some suitable constants $\hat{a}_{l \pm \delta \eta, m}, \hat{b}_{l \pm \delta \eta, m}$ and $\hat{c}_{l \pm \delta \eta, m}$.

Then, with the help of equation (33), the errors equation in its compact operator form for $l=1(1) L$, $m=1(1) M$ is given by

$$
\begin{gathered}
-\lambda^{2}\left[A_{l, m}-\frac{h^{2}}{12}\left(\frac{2 A_{x_{l, m}}^{2}}{A_{l, m}}-A_{x x_{l, m}}\right)\right] \delta_{x}^{2} \epsilon_{l, m}+\frac{k^{2}}{12 \eta^{2}}\left[\left(1-\frac{\eta h A_{x_{l, m}}}{A_{l, m}}\right)\left(\hat{E}_{l+\eta, m}-B_{l+\eta, m} \tilde{\epsilon}_{y y_{l+\eta, m}}\right)\right. \\
\left.+\left(12 \eta^{2}-2\right)\left(\hat{E}_{l, m}-B_{l, m} \tilde{\epsilon}_{y y_{l, m}}\right)+\left(1+\frac{\eta h A_{x_{l, m}}}{A_{l, m}}\right)\left(\hat{E}_{l-\eta, m}-B_{l-\eta, m} \tilde{\epsilon}_{y y_{l-\eta, m}}\right)\right] \\
=k^{4}+h^{2} k^{4}+h^{4} k^{2}
\end{gathered}
$$

The above error equation in the matrix-vector notation, is represented by the relation 


$$
\psi(\boldsymbol{u})-\psi(\boldsymbol{U})=\boldsymbol{Q} \varepsilon
$$

where $\boldsymbol{Q}=\left[Q_{p, q}\right], p, q=1(1) L M$ is the tri-block-diagonal matrix with following as the only non-zero entries.

$m=2(1) M:$

$$
\begin{aligned}
Q_{(m-1) L+\sigma,(m-2) L+\sigma-1} & =-\frac{1}{12} B_{\sigma, m}+O\left(h^{3}+k^{3}\right), \sigma=2(1) L, \\
Q_{(m-1) L+\sigma,(m-2) L+\sigma} & =-\frac{5}{6} B_{\sigma, m}+O\left(h^{3}+k^{3}\right), \sigma=1(1) L, \\
Q_{(m-1) L+\sigma,(m-2) L+\sigma+1} & =-\frac{1}{12} B_{\sigma, m}+O\left(h^{3}+k^{3}\right), \sigma=1(1) L-1,
\end{aligned}
$$

$m=1(1) M:$

$$
\begin{gathered}
Q_{(m-1) L+\sigma,(m-1) L+\sigma-1}=\frac{1}{6} B_{\sigma, m}-\lambda^{2} A_{\sigma, m}+O\left(h^{3}+k^{3}\right), \sigma=2(1) L, \\
Q_{(m-1) L+\sigma,(m-1) L+\sigma}=\frac{5}{3} B_{\sigma, m}+2 \lambda^{2} A_{\sigma, m}+O\left(h^{3}+k^{3}\right), \sigma=1(1) L, \\
Q_{(m-1) L+\sigma,(m-1) L+\sigma+1}=\frac{1}{6} B_{\sigma, m}-\lambda^{2} A_{\sigma, m}+O\left(h^{3}+k^{3}\right), \sigma=1(1) L-1,
\end{gathered}
$$

$m=1(1) M-1:$

$$
\begin{aligned}
Q_{(m-1) L+\sigma, m L+\sigma-1} & =-\frac{1}{12} B_{\sigma, m}+O\left(h^{3}+k^{3}\right), \sigma=2(1) L, \\
Q_{(m-1) L+\sigma, m L+\sigma} & =-\frac{5}{6} B_{\sigma, m}+O\left(h^{3}+k^{3}\right), \sigma=1(1) L, \\
Q_{(m-1) L+\sigma, m L+\sigma+1} & =-\frac{1}{12} B_{\sigma, m}+O\left(h^{3}+k^{3}\right), \sigma=1(1) L-1
\end{aligned}
$$

From the equations (37) and (43), we find that

$$
Q \boldsymbol{\varepsilon}=\boldsymbol{T}
$$

Therefore, for sufficiently small values of $h$ and $k$, the lower, upper and diagonal blocks have non-zero entries at the sub-diagonal, main-diagonal and sup-diagonal, provided $6 \lambda^{2}<B_{\sigma, m} / A_{\sigma, m}$. Thus, there exists a directed path connecting any two ordered pair of nodes $p$ and $q$ for the matrix $\boldsymbol{Q}$. Hence, the graph $\mathcal{G}(\boldsymbol{Q})$ of the matrix $\boldsymbol{Q}$ is strongly connected and therefore, $\boldsymbol{Q}$ is irreducible (see Varga [26]).

Let $A=\min _{p, q}\left|A_{p, q}\right|, A_{x}=\min _{p, q}\left|\left(\partial_{x} A\right)_{p, q}\right| \quad B=\min _{p, q}\left|B_{p, q}\right| \quad \hat{a}=\min _{p, q}\left|\hat{a}_{p, q}\right| \quad, \quad \hat{b}=\min _{p, q}\left|\hat{b}_{p, q}\right|, \quad \hat{c}=\min _{p, q}\left|\hat{c}_{p, q}\right|$, $p, q=1(1) L M, k=\tau h^{2}$ and $\Gamma_{q}$ be the weak row sum elements of the matrix $\boldsymbol{Q}$. Then, one obtains

$$
\begin{aligned}
& \Gamma_{1} \geq \frac{11}{12} B+O(h)>0, \\
& \Gamma_{q} \geq B+O(h)>0, \quad q=2(1) L-1, \\
& \Gamma_{L} \geq \frac{11}{12} B+O(h)>0, \\
& \Gamma_{(r-1) L+1} \geq h^{2} \tau^{2} A+\frac{h^{3}}{2} \tau^{2} \hat{a}+O\left(h^{4}\right)>0, \quad r=2(1) M-1, \\
& \Gamma_{(r-1) L+q} \geq \hat{c} \tau^{2} h^{4}+O\left(h^{5}\right) \geq 0, r=2(1) M-1, q=2(1) L-1, \text { if } \hat{c} \geq 0,
\end{aligned}
$$




$$
\begin{aligned}
& \Gamma_{(r-1) L+L} \geq h^{2} \tau^{2} A-\frac{h^{3}}{2} \tau^{2} \hat{a}+O\left(h^{4}\right)>0, \quad r=2(1) M-1, \\
& \Gamma_{(M-1) L+1} \geq \frac{11}{12} B+O(h)>0, \\
& \Gamma_{(M-1) L+q} \geq B+O(h)>0, q=2(1) L-1, \\
& \Gamma_{(M-1) L+L} \geq \frac{11}{12} B+O(h)>0,
\end{aligned}
$$

The positivity on $\Gamma_{q}$, except for the main diagonal of $\boldsymbol{Q}$ follows for the small values of $h$. Thus, if $h$ is taken to be sufficiently small, then the matrix $\boldsymbol{Q}$ is monotone. As a consequence of monotonic and irreducible behavior, $\boldsymbol{Q}^{-1}$ exists and $\boldsymbol{Q}^{-1}>0$ (see Young [29] and Henrici [8]). Let $Q_{p, q}^{-1}$ be the $(p, q)^{\text {th }}$ element of $\boldsymbol{Q}^{-1}$ and we define the matrix norm as follows

$$
\begin{array}{r}
\left\|Q^{-1}\right\|_{\infty}=\max _{p=1(1) L M}\left[\left|Q_{p, 1}^{-1}\right|+\sum_{q=2}^{L-1}\left|Q_{p, q}^{-1}\right|+\left|Q_{p, L}^{-1}\right|+\left|Q_{p,(M-1) L+1}^{-1}\right|+\sum_{q=2}^{L-1}\left|Q_{p,(M-1) L+q}^{-1}\right|\right. \\
\left.+\left|Q_{p, L M}^{-1}\right|+\sum_{r=2}^{M-1}\left(\left|Q_{p,(r-1) L+1}^{-1}\right|+\sum_{q=2}^{L-1}\left|Q_{p,(r-1) L+q}^{-1}\right|+\left|Q_{p, r L}^{-1}\right|\right)\right]
\end{array}
$$

and

$$
\boldsymbol{T}_{\infty}=\max _{l} \sum_{m=1(1) M}\left|T_{l, m}\right|=O\left(k^{4}+h^{2} k^{4}+h^{4} k^{2}\right)
$$

The matrix identity $\boldsymbol{Q}^{-1}(\boldsymbol{Q} \boldsymbol{J})=\boldsymbol{J}$, where $\boldsymbol{J}$ is $L M \times 1$ matrix with all of its elements as one. In the expanded form, we can write

$$
\sum_{q=1(1) L M} Q_{p, q}^{-1} \Gamma_{p}=1, p=1(1) L M
$$

Thus, the following bounds on the non-zero elements of the matrix $\boldsymbol{Q}^{-1}$ can be obtained with the help of Taylor's expansions. $p=1(1) L M$ :

$$
\begin{aligned}
& Q_{p, 1}^{-1} \leq \frac{1}{\Gamma_{1}} \leq \frac{12}{11 B}+O(h), \\
& \sum_{q=2}^{L-1} Q_{p, q}^{-1} \leq \frac{1}{\min _{q=2(1) L-1} \Gamma_{q}} \leq \frac{1}{B}+O(h), \\
& Q_{p, 1}^{-1} \leq \frac{1}{\Gamma_{L}} \leq \frac{12}{11 B}+O(h), \\
& \sum_{r=2}^{M-1} Q_{p,(r-1) L+1}^{-1} \leq \frac{1}{\min _{r=2(1) M-1} \Gamma_{(r-1) L+1}} \leq \frac{1}{h^{2} \tau^{2} A}-\frac{\hat{a}}{2 h \tau^{2} A^{2}}+O\left(h^{\xi}\right) Q \xi \geq, \\
& \sum_{r=2}^{M-1 L-1} \sum_{q=2} Q_{p,(r-1) L+q}^{-1} \leq\left\{\begin{array}{l}
\sum_{q=1}^{L M} Q_{p, q}^{-1} \Gamma_{q}=1, \hat{c}=0 \\
\frac{1}{\min _{q=2(1) L-1} \Gamma_{(r-1) L+q}} \leq \frac{1}{r^{4} \tau^{2} \hat{c}(1) M-1}-\frac{(\eta-1)\left(f A-\hat{a} A_{x}\right)}{12 h^{2} \tau^{2} \eta \hat{c} A^{2}}+O\left(h^{\xi}\right), \xi \geq 0, \hat{c} \neq 0
\end{array}\right.
\end{aligned}
$$




$$
\begin{aligned}
& \sum_{r=2}^{M-1} Q_{p, r L}^{-1} \leq \frac{1}{\min _{r=2(1) M-1} \Gamma_{r L}} \leq \frac{1}{h^{2} \tau^{2} A}+\frac{\hat{a}}{2 h \tau^{2} A^{2}}+O\left(h^{\xi}\right), \xi \geq 0 \\
& Q_{p,(M-1) L+1}^{-1} \leq \frac{1}{\Gamma_{(M-1) L+1}} \leq \frac{12}{11 B}+O(h) \\
& \sum_{q=2}^{L-1} Q_{p,(M-1) L+q}^{-1} \leq \frac{1}{B}+O(h) \\
& Q_{p, M L}^{-1} \leq \frac{1}{\Gamma_{L M}} \leq \frac{12}{11 B}+O(h)
\end{aligned}
$$

Thus, we obtain

$$
h^{8} \boldsymbol{Q}_{\infty}^{-1} \leq\left\{\begin{array}{c}
\frac{h^{4}}{\hat{c} \tau^{2}}+\frac{h^{6}}{12 \eta \tau^{2} \hat{c} A^{2}}\left(A_{x}(\eta-1) \hat{a}+A(23 \eta+1)\right)+O\left(h^{8}\right), \hat{c}>0 \\
O\left(h^{4}\right), \hat{c}=0
\end{array}\right.
$$

In combination of the above inequalities and equation (44), we conclude that

$$
\boldsymbol{\varepsilon}_{\infty} \leq \boldsymbol{Q}_{\infty}^{-1} \bullet \boldsymbol{T}_{\infty} \leq O\left(h^{4}\right)
$$

This implies that, the solution error $(\varepsilon)$ occurred due to finite difference replacement vanishes as $h \rightarrow 0$. We conclude the above results as a following theorem that ensures the existence of numerical solution.

Theorem 1: The non-polynomial spline finite difference method (30) is accurate for the numerical solution of EBVPs (11) with Dirichlet boundary conditions and converges for sufficiently small values of $h$ and $6 \lambda^{2}<B_{l, m} / A_{l, m}$, provided $\partial_{U} G \geq 0$ and $\partial_{U_{x}} G, \partial_{U_{y}} G$ are bounded. Here, the condition $\partial_{U} G \geq 0$ corresponds to $\hat{c} \geq 0$.

\section{Numerical Verifications}

To illustrate the application of proposed non-polynomial spline finite difference scheme, we have obtained the approximate solution values and compared them with the exact solution values for quasilinear EBVPs occurring in the various physical applications. The corresponding polynomial spline difference equation is obtained as a limiting case of hyperbolic function frequency parameter $(\omega)$ to zero. The accuracy of solution values is compared using root mean square errors and maximum absolute value defined by the relations

$$
\|\varepsilon\|_{2}=\left(\frac{1}{L M} \sum_{l=1}^{L} \sum_{m=1}^{M}\left|U_{l, m}-u_{l, m}\right|^{2}\right)^{1 / 2}, \quad\|\varepsilon\|_{\infty}=\max _{l, m}\left|U_{l, m}-u_{l, m}\right| .
$$

The computational order of convergence is presented, showing close resemblance with the theoretical error estimation. The metrics of orders are defined by formula

$$
\Theta_{\infty}=\log _{2}\left[\frac{\|\varepsilon\|_{\infty}^{(L, M)}}{\|\varepsilon\|_{\infty}^{(2 L+1,2 M+1)}}\right], \quad \Theta_{2}=\log _{2}\left[\frac{\|\varepsilon\|_{2}^{(L, M)}}{\|\varepsilon\|_{2}^{(2 L+1,2 M+1)}}\right] .
$$

In order to illustrate the computational convergence, we have taken values of mesh ratio parameter $\tau=20$. The solutions are obtained by Gauss-Seidel and Newton-Raphson method. The error tolerance is taken as $10^{-10}$ and in non-linear problems the initial guess is taken as a zero vector (Hageman and Young [7]). As a test procedure, boundary conditions are obtained from the analytical solution. The 
algebraic calculations are obtained in the Maple environment; while numerical computations are executed with $\mathrm{C}$ programming.

Example 5.1: (Babich et. al. [3]) Consider the Tricomi equation describing near-sonic flows of gas

$$
y \partial_{x}^{2} U+\partial_{y}^{2} U=0,(x, y) \in(0,1) \times(0,1)
$$

The analytical solution is given by $U(x, y)=3 x^{2}-y^{3}+x^{3}-x y^{3}+6 y x^{2}-y^{4}$. The accuracy of solution values in terms of maximum absolute errors and root mean square errors along with their convergence order is computed and shown in Table 1. The theoretical order of accuracy has close resemblance with the computed results.

Example 5.2: (Polyanin et al. [21]) Consider the Graetz-Nusselt equation, which is governing steady state heat exchange in a laminar fluid flow with parabolic velocity profile in a plane channel

$$
\partial_{x}^{2} U+\partial_{y}^{2} U=P_{e}\left(1-y^{2}\right) U_{x},(x, y) \in(0,1) \times(0,1)
$$

The analytical solution is given by $U(x, y)=12 x+P_{e} y^{2}\left(6-y^{4}\right)+1$ and the quantity $P_{e}$ denotes the Peclet number. Errors of the exact and approximate solution for $P_{e}=100$ are given in Table 2 . Comparing the results of non-polynomial spline with $\eta \neq 1$, it has been observed that the iteration number (Itr) and consequently, the computing time to achieve the desired accuracy is much less compared to polynomial spline with $\eta=1$. The graphical solutions on the domain $(-2,2) \times(-2,2)$ has been shown in Figure 1, with $h=1 / 40, \eta=0.5$ and $P_{e}=100$.

Example 5.3: (Kozlov et al. [14]) Consider the stationary Khokhlov-Zabolotskaya quasi-linear EBVPs, which arises in acoustics and mass transfer theory

$$
\partial_{x}^{2} U+\partial_{y}\left(U \partial_{y} U\right)=0, \quad(x, y) \in(0,1) \times(0, \pi)
$$

The analytical solution is given by $U(x, y)=-1+\sqrt{1+x+y}$. The accuracy of solutions are obtained in Table 3 for various values of $h$ in case of non-polynomial spline finite difference scheme.

Example 5.4: (Polyanin et al. [22]) Consider the quasi-linear EBVPs occurring in the theory of combustion

$$
\partial_{x}\left(U \partial_{x} U\right)+\partial_{y}\left(U \partial_{y} U\right)=4 e^{2(x+y)},(x, y) \in(0,1) \times(0,1)
$$

The analytical solution is given by $U(x, y)=e^{x+y}$. The accuracy in the solutions are obtained for small as well as large values of spline parameter $\omega$ in Table 4 using various values of $h$. The iteration number (Itr) and consequently the computing time shows tremendous improvement in the case of nonpolynomial spline and $\eta \neq 1$, while order and accuracy being almost unchanged.

\section{Concluding Remarks}

In this article, we have solved two-dimensional quasi-linear EBVPs by means of finite difference approximations having compact character and one dimensional non-polynomial spline basis. Importance of the parameter $\eta$ with non-unit value and spline parameter $\omega$ being not small has been illustrated by the help examples. The proposed method shows superiority over cubic polynomial spline scheme in terms of iteration number and consequently the computing time, and it is capable of achieving accuracy of fourth order. The method is convergent and can be extended to three-dimensional non-linear EBVPs. 


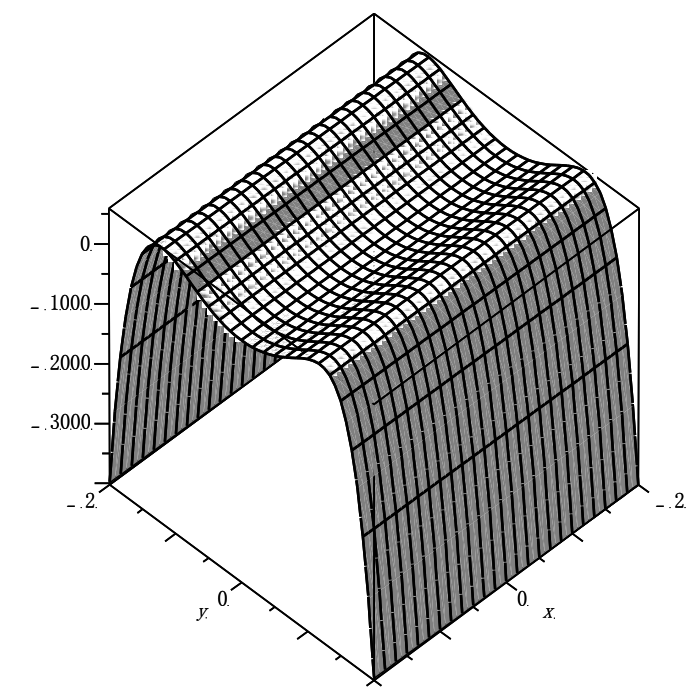

Fig. 1 Numerical solution to Example 5.2

Table 1: Solution errors and computational order of convergence for example 5.1.

\begin{tabular}{|r|c|c|c|c|c|c|}
\hline$h$ & $\omega$ & $\eta$ & $E_{\infty}$ & $E_{2}$ & $O_{\infty}$ & $O_{2}$ \\
\hline $1 / 10$ & 5 & 0.8 & $7.59 \mathrm{e}-03$ & $5.17 \mathrm{e}-03$ & --- & --- \\
\hline $1 / 20$ & 5 & 0.8 & $4.87 \mathrm{e}-04$ & $2.92 \mathrm{e}-04$ & 4.0 & 4.1 \\
\hline $1 / 40$ & 5 & 0.8 & $3.06 \mathrm{e}-05$ & $1.77 \mathrm{e}-05$ & 4.0 & 4.0 \\
\hline
\end{tabular}

Table 2: Solution errors and computational order of convergence for example 5.2.

\begin{tabular}{|r|c|c|c|c|c|c|c|}
\hline$h$ & $\omega$ & $\eta$ & $I t r$ & $E_{\infty}$ & $E_{2}$ & $O_{\infty}$ & $O_{2}$ \\
\hline $1 / 10$ & 0 & 1 & 58 & $1.26 \mathrm{e}-01$ & $6.45 \mathrm{e}-02$ & --- & --- \\
\hline $1 / 20$ & 0 & 1 & 95 & $8.73 \mathrm{e}-03$ & $4.07 \mathrm{e}-03$ & 3.8 & 4.0 \\
\hline $1 / 40$ & 0 & 1 & 841 & $5.48 \mathrm{e}-04$ & $2.50 \mathrm{e}-04$ & 4.0 & 4.0 \\
\hline $1 / 10$ & 20 & 0.36 & 19 & $1.44 \mathrm{e}-01$ & $6.81 \mathrm{e}-02$ & --- & --- \\
\hline $1 / 20$ & 20 & 0.21 & 80 & $9.21 \mathrm{e}-03$ & $4.16 \mathrm{e}-03$ & 4.0 & 4.0 \\
\hline $1 / 40$ & 30 & 0.10 & 765 & $5.57 \mathrm{e}-04$ & $2.54 \mathrm{e}-04$ & 4.0 & 4.0 \\
\hline
\end{tabular}

Table 3: Solution errors and computational order of convergence for example 5.3.

\begin{tabular}{|r|c|c|c|c|c|c|}
\hline$h$ & $\omega$ & $\eta$ & $E_{\infty}$ & $E_{2}$ & $O_{\infty}$ & $O_{2}$ \\
\hline $1 / 10$ & 10 & 1 & $1.95 \mathrm{e}-04$ & $8.29 \mathrm{e}-05$ & --- & --- \\
\hline $1 / 20$ & 10 & 1 & $2.09 \mathrm{e}-05$ & $6.72 \mathrm{e}-06$ & 3.2 & 3.6 \\
\hline $1 / 40$ & 10 & 1 & $1.37 \mathrm{e}-06$ & $4.14 \mathrm{e}-07$ & 3.9 & 4.0 \\
\hline
\end{tabular}

Table 4: Solution errors and computational order of convergence for example 5.4.

\begin{tabular}{|r|c|c|c|c|c|c|c|}
\hline$h$ & $\omega$ & $\eta$ & Itr & $E_{\infty}$ & $E_{2}$ & $O_{\infty}$ & $O_{2}$ \\
\hline $1 / 10$ & 0.0001 & 1.0 & 178 & $3.72 \mathrm{e}-03$ & $2.53 \mathrm{e}-03$ & --- & --- \\
\hline $1 / 20$ & 0.0001 & 1.0 & 609 & $2.43 \mathrm{e}-04$ & $1.43 \mathrm{e}-04$ & 3.9 & 4.1 \\
\hline $1 / 40$ & 0.0001 & 1.0 & 2418 & $1.52 \mathrm{e}-05$ & $8.66 \mathrm{e}-06$ & 4.0 & 4.0 \\
\hline $1 / 10$ & 300 & 1.0 & 103 & $3.58 \mathrm{e}-03$ & $2.43 \mathrm{e}-03$ & --- & --- \\
\hline
\end{tabular}




\begin{tabular}{|c|c|c|c|c|c|c|c|}
\hline $1 / 20$ & 300 & 1.0 & 439 & $2.09 \mathrm{e}-04$ & $1.23 \mathrm{e}-04$ & 4.1 & 4.3 \\
\hline $1 / 40$ & 300 & 1.0 & 2072 & $7.86 \mathrm{e}-06$ & $4.49 \mathrm{e}-06$ & 4.7 & 4.8 \\
\hline $1 / 10$ & 300 & 0.8 & 98 & $3.49 \mathrm{e}-03$ & $2.37 \mathrm{e}-03$ & --- & --- \\
\hline $1 / 20$ & 300 & 0.8 & 413 & $1.81 \mathrm{e}-04$ & $1.07 \mathrm{e}-04$ & 4.3 & 4.5 \\
\hline $1 / 40$ & 300 & 0.5 & 1739 & $1.09 \mathrm{e}-05$ & $6.22 \mathrm{e}-06$ & 4.1 & 4.1 \\
\hline
\end{tabular}

\section{References}

1. Andrew, V.K., Kaptsov, O.V., Pukhnachov, V.V., Rodionov, A.A. Applications of group theoretical methods in hydrodynamics. Kulwer, Dordrecht (1999)

2. Aziz, I., Siraj-ul-Islam, Šarler, B. "Wavelets collocation methods for the numerical solution of elliptic BV problems." Appl. Math. Model. 37, 676--694 (2013)

3. Babich, V.M., Kapilevich, M.B., Mikhlin, S.G. Linear equations of mathematical physics. Nauka, Moscow (1964)

4. Deka, B. "Finite element methods with numerical quadrature for elliptic problems with smooth interfaces." J. Comput. Appl. Math. 234, 605--612 (2010)

5. Evans, L.C. Partial differential equations. American Mathematical Society Providence, Rhode Island (2002)

6. Gopal, V., Mohanty, R.K., Saha, L.M. "A new high accuracy non-polynomial tension spline method for the solution of one dimensional wave equation in polar co-ordinates." J. Egyptian Math. Soc. 22, 280--285 (2014)

7. Hageman, L.A., Young, D.M. Applied iterative methods. Dover Publication, New York (2004)

8. Henrici, P. Discrete variable methods in ordinary differential equations. Wiley, New York (1962)

9. Hu, H.Y. "Radial basis collocation methods for elliptic boundary value problem." Comput. Math. Appl. 50, 289--320 (2005)

10. Ibragimov, N.H. Transformation groups applied in mathematical physics. D. Reidel Publ., Dordrecht (1985)

11. Jha, N., Mohanty, R.K. "Quintic hyperbolic non-polynomial spline and finite difference method for nonlinear second order differential equations and its application." J. Egyptian Math. Soc. 22, 115--122 (2014)

12. Jha, N. "High order accurate quintic non-polynomial spline finite difference approximations for the numerical solution of non-linear two point boundary value problems." Int. J. Model. Simul. Sci. Comput. 5, 1--16 (2014)

13. Khan, A. "Parametric cubic spline solution of two point boundary value problems." Appl. Math. Comput. 154, 175--182 (2004)

14. Kozlov, V.V. Symmetries, topology and resonances in Hamiltonian mechanics. Izd-vo Udmurtskogo Gos. Universiteta, Izhevsk (1995)

15. Lambert, J.D. Computational methods in ordinary differential equations. Wiley (1973)

16. Lynch, R.E., Rice, J.R. "High accuracy finite difference approximation to solutions of elliptic partial differential equations." Proc. Natl. Acad. Sci. 75, 2541--2544 (1978)

17. LeVeque, R.J. Finite difference methods for ordinary and partial differential equations: steady state and time-dependent problems. SIAM, (2007) 
18. Mohanty, R.K., Sachdev, P.L., Jha, N. "An O $\left(h^{4}\right)$ accurate cubic spline TAGE method for non-linear singulat two point boundary value problems." Appl. Math. Comput. 158, 853--868 (2004)

19. Mohanty, R.K., Jain, M.K., Dhall, D. "High accuracy cubic spline approximation for two dimensional quasi-linear elliptic boundary value problems." Appl. Math. Model. 37, 155--171 (2013)

20. Polyanin, A.D., Handbook of linear partial differential equations for engineers and scientists, CRC press (2010)

21. Polyanin, A.D., Kutepov, A.M., Vyazmin, A.V., Kazenin, D.A. Hydrodynamics, Mass and heat transfer in chemical engineering. Gordon \& Breach Sci. Publ., London (2001)

22. Polyanin, A.D., Zaitsev, V.F. Handbook of nonlinear partial differential equations, Chapman \& Hall/CRC Press (2003)

23. Rashidinia, J., Mohammadi ,R., Jalilian, R. "Spline methods for the solution of hyperbolic equation with variable coefficients." Numer. Meth. Part. D. E. 23, 1411--1419 (2007)

24. Saldanha, G., Ananthakrishnaiah, U. "A fourth-order finite difference scheme for twodimensional nonlinear elliptic partial differential equations." Numer. Meth. Part. D. E. 11, 33-$40(1995)$

25. Siraj-ul-Islam, Tirmizi, I.A. "A smooth approximation for the solution of special non-linear third-order boundary-value problems based on non-polynomial spline." Int. J. Comput. Math. 83(4), 397--407 (2006)

26. Varga R.S. Matrix Iterative Analysis. Springer Series in Computational Mathematics. Springer Berlin, Germany (2000)

27. Weiser, A., Wheeler, M. F. "On convergence of block-centered finite differences for elliptic problems." SIAM J. Numer. Anal. 25, 351--375 (1988)

28. Young, D.L., Chang, T.J., Eldho, T.I. "The Riemann complex boundary element method for the solutions of two-dimensional Elliptic equations." Appl. Math. Model. 26, 893--911 (2002)

29. Young, D.M. Iterative solution of large linear systems. Courier Dover Pub., 2013.

30. Zhao, J., Zhang, T., Corless, R.M. "Convergence of the compact finite difference method for second-order elliptic equations." Appl. Math. Comput. 182, 1454--1469 (2006) 